\title{
Application of Nanometer Materials in Protein Separation
}

\author{
Zhen-Rong LIN ${ }^{b}$, Yun-Yun XU ${ }^{a, *}$ and Tao ZHANG ${ }^{b}$ \\ (Airforce Logistics College, Xuzhou 221000,China) \\ axuyunyun713@163.com, b517yy@sohu.com \\ *Corresponding author
}

Keywords: nanometer material, protein separation, application, review

\begin{abstract}
The technology of quantitative separation of proteins is of great importance to drugtesting and pharm aceutical engineering, and it has become one of the hotspots in biochem icalengineering and protein analysis. With many special properties different from those of bulk materials, nanometer materials have been widely applied in chemical industry, biology, medicine, space industry and so on, and they are considered to be the most promising materials in 21st century. This review mainly focuses on the application of nanometer materials in protein separation in the past decades, covering nonmetal oxide, nonmetal, metal oxide, metal, nanometerpolymer, and nanoscale composites. The advantages and disadvantages of these nanometer materials and their application status are summarized. Besides, the application prospect of nanometer materials in protein obilization and separation is proposed.
\end{abstract}

\section{Introduction}

In recent years, many biological functions of materials were prepared by surface modification and widely used in medicine [1-8], biology and other fields, especially in the separation of various proteins[9-10]. Protein is an important component of all living organisms, and it is the material basis of all life. All the metabolic activities of the living body, including growth, development and reproduction and so on, are related to the activity and metabolism of protein. The cells that comprise the organism include different kinds of proteins, and each of which has a different structure and function.It needs a single analysis and repair as the disassembly and assembly of machine components to reveal the structure and function of a protein from its essence. Therefore, it is of great significance to reveal the phenomenon and essence of a certain life by using the integrated biochemical method to separate a particular protein.

Proteins are usually mixed in the tissues and cells, and different types of cells which contain thousands of different proteins. To extract proteins from such a hybrid and numerous proteins is a difficult task, therefore, the separation and purification of total goal should be to increase the purity of the product, maintain the biological activity of protein separation, and reduce separation process in the cost of consumption to achieve high efficiency and low consumption with high quality. Protein separation and purification is a hot research topic in biochemical engineering and protein analysis. In recent years, the nano materials as the carrier, the surface of the functional treatment to get a new type of biological function of the carrier material, can be widely used in protein separation and purification. The main methods of protein separation and purification are precipitation[11], chromatography[12], electrical method[13], centrifugal method[14], membrane separation technology[15].

Chromatography is an efficient method to isolate protein, and it is widely used in the experiment of nano materials as a carrier. The chromatography first began at the beginning of the 20 century. It has been developed rapidly since 1950. It used different materials in different phases of the selective distribution, and the mixture of the stationary phase was eluted. The different substances in the mixture will move at different speeds along the fixed phase, and finally reach the separation effect. According to the physical or physical chemical properties of separation, chromatography is divided into ion exchange chromatography, molecular exclusion chromatography, affinity chromatography, adsorption chromatography and so on. Chromatography has the following 
advantages: high separation efficiency, rapid separation speed, high detection sensitivity, less sample consumption and good selectivity.In addition to simultaneous multicomponent analysis and can realize automatic operation from sampling to data processing. But the ability of qualitative analysis of chromatography is poor, and it is generally to be combined with other analytical techniques which have the ability of qualitative analysis.

\section{Application of nano materials in protein separation and purification}

\section{Non-metal oxide nanomaterials}

\section{Nano-SiO2}

In the non metal oxide nano materials, nano-SiO2 is a kind of commonly used materials, which has the advantages of no toxicity, no smell, good biocompatibility, stable property, strong adsorption ability and so on. The surface has a large number of hydroxyl groups, for a variety of chemical reactions and biological molecules in the surface of the combination provides a convenient condition, in the protein separation and purification is playing an increasingly important role[16-18]. Xue Yan Zou modified amino on the surface of the nano silica[19], and the GSH group was bonded with glutaraldehyde as the crosslinking agent .By using specific adsorption of glutathione and glutathione S-transferase enzyme, glutathione S-transferase is separated from mixed protein. In order to improve the grafting ratio of the active groups on the silica surface, the thiol group of silica was synthesized by one step method. And on the surface of the biological function of glutathione $\mathrm{S}$ transferase separation, the separation effect is good. This method provides a new idea for the study of the biological function of silica. In order to improve the grafting ratio, Li et al.[20] used a silane coupling agent to prepare nano $\mathrm{SiO} 2-\mathrm{SH}$ microspheres, and $\mathrm{SiO} 2$ was prepared by the surface bonding of GSH molecules.

\section{Mesoporous molecular sieve}

Mesoporous molecular sieve is a kind of inorganic porous materials which with narrow pore size, highly regular and orderly structure and high specific surface area. It has good application prospects in the fields of catalytic conversion, adsorption separation, drug delivery and release, preparation of nano materials and so on. In 1997, Raimond was used to separate the glass capillary column on the mesoporous molecular sieve M41-S by sol gel technique, which was used as a gas chromatographic separation. Lei Jie in the mesoporous molecular sieve SBA-15 surface grafting with diethylaminoethyl (DEAE) made a weak anion exchange chromatography agent deae-sba-15. The protein has the function of molecular sieve and ion exchange, and can separate and purify the main allergen Derf2 protein of the dust mite in the metabolic culture medium.And Derf2 still has the activity of allergen.

\section{Non-metal nano materials}

In non metallic simple substance nano materials, carbon nanotubes is the carrier of more researches. It can be combined with protein amino and carboxyl functional groups prepared several protein function of biological materials, which applied to the analysis of biological samples, electrochemical reaction mechanism, metal particles and organic pollutants detection. The multi walled carbon nanotubes were formed by carboxyl group to form active ester, and the amino reaction between the active ester and iron protein and bovine serum protein formed amide bond by Jiang et al. Thus, the iron protein and the bovine serum protein are bonded to the surface of the carbon nanotubes. This process avoids the interaction between protein molecules, and provides an effective way to connect carbon nanotubes with biological molecules.Mubarak using carbon nanotubes as a carrier, successfully isolated milk protein from purified skim , electrophoresis buffer $\mathrm{pH}$ and ionic strength are optimized and the carbon nanotubes can also as a hydrophobic interaction chromatography of effective medium.Zhang using mesoporous silica carbon nanotubes to separate the cell pigment (Cyt), bovine serum albumin (BSA), lysozyme (Lyz) from mixed solution. When the isoelectric point ( $\mathrm{pH}$ 9.6) reached the cytochrome Cyt, the adsorption capacity of $\mathrm{C}$ reached the 
maximum, while BSA and Lyz were almost not adsorbed at the isoelectric point of BSA and Lyz.The adsorbed Cyt can be re released in a buffer solution of high ionic strength, and the mesoporous silica carbon nanotubes can be recycled after treatment.

\section{Metal oxide nano materials}

Nano $\mathrm{Fe}_{2} \mathrm{O}_{3}, \mathrm{Fe}_{3} \mathrm{O}_{4}, \mathrm{TiO}_{2}, \mathrm{ZrO}, \mathrm{Al}_{2} \mathrm{O}_{3}, \mathrm{~V}_{2} \mathrm{O}_{5}$ and other metal oxide nano materials are potential vector of biological materials with good biocompatibility. These nanoparticles can be directed to some groups of proteins, so that the biological molecules can be in their surface orientation and orientation rules.

\section{Metal nano materials}

Nano gold, silver, copper and other metallic nano particles with specific surface area and strong adsorption ability, good biocompatibility, the biological molecules fixed on the surface strongly by biological sensors or other devices.And it has also been widely used in the purification of protein.Due to the surface effect and quantum size effect, covalent coupling through electrostatic adsorption or sulfhydryl modification sites, gold nanorods surface easy to be key molecular probe antibody and oligonucleotide.Yu on the surface of didodecyl dimethyl ammonium bromide (DDAB) covering the nano gold particles, for the separation of acidic and basic proteins. The method has the advantages of simple operation, high separation efficiency, and good reproducibility.

\section{Nano polymer}

The nano polymer has a lot of different atoms and molecules, but also different from the nature of the macro.Widely used in high-tech field, its application has penetrated into chemistry, biology, optics, magnetics, mechanics, materials science fields.A single layer of chlorine - methylated polystyrene, is adsorbed on the inner wall of the capillary, which is used to separate the protein or polypeptide by Kleindienst[21].As the carrier of anodic aluminum oxide film (AAO), the molecularly imprinted polymer was synthesized by using the protein as the imprinted molecule, which solved the problem that the imprinted protein was difficult to be eluted by Li Yong[22].The polymer of bovine hemoglobin (BSA) has a specific binding ability, and has a higher adsorption capacity.In addition,because of its rich resources, easy surface modification and good biocompatibility,chitin and chitosan is also widely used in the BSA, chitin binding protein, condensation of hormone and enzyme protein separation and purification in recent years .

\section{Nano composite materials}

With the development of nano technology, single nano materials can not meet the demand of the market. In recent years, many scholars have been committed to the synthesis and application of nano composite materials. Magnetic particles in the presence of the external magnetic field can be directed movement, and has an important application in the biochemical separation. Base on the surface of silica nanotubes modified gold nanoparticles, and gold nanoparticles for the bridge will connect the nanotube surface sulfhydryl and cysteine thiol, so as to realize the separation of cysteine and cysteine fragments of other proteins containing.

\section{Discussion}

With the deep study of nano materials, there will be more and more nano materials are biological function for protein purification and other biomedical fields, and eventually become the main force of the protein separation, bio pharmaceuticals, medical diagnosis, medical imaging and other. However, many of the current applications are still relatively preliminary, only limited to the laboratory, many technologies to achieve industrial production have a certain distance, and there are still many problems need to be resolved and in-depth study. 


\section{References}

[1]BIENER M, BIENER J, WICHMANN A, et a1. ALD functionalized nanoporous gold: thermal stability, mechanical properties, and catalytic activity[J]. Nano Lett, 2011, 11: 3085-3090.

[2]HSU S, TSENG H J, HUNG H S, et a1. Antimicrobial activities and cellular responses to natural silicate clays and derivatives modified by cationic alkylamine salts I-J]. ACS Appl Mater Inter, 2009, 1(11): 2556-2564.

[3]PANYAM J, LABHAsETwAR V. Biodegradable nanoparticles for drug and gene delivery to cells and tissue[J]. AdvDrug Delivery Rev, 2012,55(3):329-347.

[4] BATES M, CLOETE T E. The potential of nanofihers and nanohiocides in water purification[J]. Crit Rev Microbial, 2010,36(1):68-81.

[5]PARK J J, LEE J G, KIM D Y. Antibacterial and water purification activities of self-assembled Honeycomb structure of aerosol deposited titania film [J]. Environ Sci Technol, 2012,46(22): 12510-12518.

[6] LOU X W, DENG D, LEE J Y. Self supported formation of needlelike Co3 O4 nanotubes and their application as lithium ion battery electrodes[J]. Adv Mater, 2008,20(2):258-262.

[7]DARIUS J, MEYER F P, SABEL B A, et a1. Influence of nanoparticles on the brain to serum distribution and the metabolism of valproic acid in mice[J]. J Pharm Pharmacol,2000,52(9): 1043-1047.

[8]MOTORIN Y, HELM M. RNA stabilization by modified nucleotides[J]. Biochemistry, 2010, 49(24):4934-4944.

[9]GODULA K, UMBEL M L, RABUKA D. Control of the molecular orientation of membrane-anchored biomimetic glycopolymers[J]. J Am Chem Sac,2009,131(29):10263-10268.

[10]CHRISTMAN K L, BRAYER R M, SCHOPF E. Protein nanopatterns by oxime bond formation[J]. Langmuir, 2011, 27(4):1415- 1418.

[11]KAY R, BARTON C, RATCLIFFE L, et a1. Enrichment of low molecular weight serum proteins using acetonitrile precipitat ion form assspectrometry based proteomic analysis I-J]. Rapid Commun Mass SP, 2008, 22: 3255-3260.

[12]DING T, XU J, LI J, et a1. Determination of melamine residue in plant origin protein powders using high performanceliquid chromatography_diode array detection and high performance liquid chromatography-electrospray ionization tandemmass spectrometry[J]. Chin J Chromatogr, 2008, 26(1):6-9.

[13] MALTAS E, OZMEN M, VURAL H C, et a1. Immobilization of albumin on magnetite nanoparticles[J]. Mater Lett,2011, 65(23 / 24): 3499-3501.

[14]LAWRENCE J E, STEWARD G F. Purification of viruses by centrifugation[J]. Manual of Aquatic Viral Ecol ASLO, 2010,17:166-181.

[15]JANSON J C. Protein purification: Principles, high resolution methods, and applications[M]. 3rd ed. New York: wiley, 2011:281- 300.

[16]LIN Yuchun, LIANG Mingren, LIN Yuchen, et a1. Specifically and reversibly immobilizing proteins / enzymes to nitriolotriacetic_acid—modified mesoporous silicas through histidine yags for purification or catalysis[J]. Chem Eur J, 2011,17(46):13059-13067.

[17]QIN Weidong. Silica nanoparticles as pseudostationary phase for protein separation[J]. ElectrophOresis, 2007,28(17):3017-3023. 
[18]KATIYAR A, PINTO N G. Visualization of size selective protein separations on spherical mesoporous silicates[J]. Small,2006,2(5):644-648.

[19] Xue Yan Zou,Binjie Li,Shulian Li. Surface modification of SiO nano particles and separation of glutathione from glutathione[J]. Journal of Henan University, 2011,41(4):366-369.

[20]Li Binjie,Zhao Yanbao, et a1. Biofunctionalization of silica microspheres for protein separation[J]. MatSci Eng C,2013,33:2595-2600.

[21] Kleindienst A. Separation of proteins or polypeptides from single layer of polystyrene nano materials[J]. Small,2011,23(15):2614-2618.

[22]Li Yong,Wang YuMei,Chang ChunRong. Anodic aluminum oxide film as a carrier for the synthesis of molecularly imprinted polymer[J]. Journal of Lanzhou University, 2012,26(13):257-260. 